$37 \%$ increase in the inclusion of problem lists and $12 \%$ increase in medication list inclusion.

Conclusion Effective communication is an integral part of clinical medicine. According to the modified SAIL assessment tool letters were not effectively communicating clinic details to primary care physicians posing a threat to patient care at the interface of paediatric primary and secondary care. Regular education and a pre designed layout positively impacts the quality of our written communication. Further steps need to be taken to ensure the continuity of standards in a centre where NCHDs rotate frequently.

\section{GP122 IS TREATMENT WITH ATROPINE 0.01\% EYEDROPS SAFE TO PREVENT THE PROGRESSION OF CHILDHOOD MYOPIA?}

\begin{abstract}
1,2 Jose Manuel Siurana, ${ }^{1}$ Georges Akel*, 'Lluis Cavero, ${ }^{1}$ Anna Mones, ${ }^{1}$ Ezequiel Anabalon, ${ }^{1}$ Paula Sol Ventura. ${ }^{1}$ Fundacio Hospital de Nens, Barcelona, Spain; ${ }^{2}$ Autonomous University, Barcelona, Spain
\end{abstract}

\subsection{6/archdischild-2019-epa.187}

Introduction In 2016 the effectiveness of atropine eyedrops in preventing the progression of myopia in children was confirmed. Atropine produces parasympathetic inhibition because avoids the acetylcholine fixation. Atropine's main indication is the cardiac stimulation in vagal bradycardia. Its principal cardiovascular side effects are: palpitations, tachycardia and atrial arrhythmias. A paradoxical bradycardia has been documented when atropine is administered at lower doses $(<0.1 \mathrm{mg})$. However, this concept has now been refuted. The last guidelines of the American Heart Association for Cardiopulmonary Resuscitation recommend to eliminate the minimum dose restriction of atropine during emergency intubation (class IIb). The main reluctance of parents to use atropine eyedrops in the control of myopia of their children is the fear of the cardiac effect.

Objective To evaluate the presence of cardiovascular changes after treatment with $0.01 \%$ atropine eyedrops administered to reduce the progression of myopia in children.

Material and methods Prospective observational study in 54 patients at the Nens Hospital Foundation in Barcelona (Spain) between 2016-2018. Patients received one drop of atropine $0.01 \%$ in each eye (total dose: $0.01 \mathrm{mg}$ ) with one minute of tear duct occlusion. Two analyzes were performed: before starting atropine and after 3 months of treatment. The parameters assessed were: somatometric data (weight and height), constants (heart rate and blood pressure), electrocardiographic data (P wave axis, PR segment and arrhythmias) and ultrasound data (left ventricle tele-diastolic diameter).

Results The average age was 10.2 years, with a higher percentage of women $(68.5 \%$ vs $31.5 \%)$. Only one patient presented symptoms of palpitations and there was only one case with arrhythmias. It was an 8 years old girl who presented premature atrial beats. There were no significant differences in the studied variables, with the exception of heart rate. The mean heart rate decreased from $79.4 \mathrm{bpm}$ to $75.3 \mathrm{bpm} \mathrm{(p}$ $<0.05)$.

Conclusion The use of atropine $0.01 \%$ eyedrops to prevent the progression of childhood myopia is cardiologically safe. Daily use of very low doses of atropine $(0.01 \mathrm{mg})$ over a period of 3 months results in a significant decrease in heart rate.

\section{GP123 ALTERED SYSTEMIC INFLAMMATORY RESPONSE IN PAEDIATRIC MILD TRAUMATIC BRAIN INJURY}

${ }^{1,2}$ Emer Ryan*, ${ }^{1}$ Eimear Duff, ${ }^{1}$ Dean Huggard, ${ }^{3}$ Mark Bates, ${ }^{4}$ Derek G Doherty, ${ }^{4}$ Ashanty M Melo, 1,2,5,6Eleanor Molloy. 'Discipline of Paediatircs, Department of Medicine, Trinity College, Dublin, Ireland; ${ }^{2}$ Tallaght University Hospital, Dublin, Ireland; ${ }^{3}$ Trinity Translational MedicDepartment of Surgery, Trinity Translational Medicine Institute, Trinity College, Dublin, Ireland; ${ }^{4}$ Trinity Translational Medicine Institute, Trinity College, Dublin, Ireland; ${ }^{5}$ National Childrens Reasrhc earch Centre, Dublin, Ireland; ${ }^{6}$ The Coombe Women's Hospital, Dublin, Ireland

\subsection{6/archdischild-2019-epa.188}

Aims To evaluate systemic inflammation in TBI by exploration of the inflammasome pathway, a component of the innate immune system that regulates and induces inflammation. We examine the pathway at baseline in TBI compared with healthy control children, and in vitro in response to both LPS stimulation and melatonin therapy. Melatonin has protective effects against NLRP3 inflammasome activation and has therapeutic implications.

Methods Whole blood was sampled from children with TBI $(n=10)$ within 24 hours of injury and compared to healthy age-matched controls $(n=8)$ at baseline, following stimulation with bacterial endotoxin (LPS) $(10 \mathrm{ng} / \mathrm{ml})$ and melatonin treatment $\left(10^{-3} \mathrm{M}\right)$.

Granulocytes were delineated as CD66b+ and FSC, SSC-A. Measurements of mean channel fluorescence (MCF) of CD11b and TLR4 expression on FACS Canto II were recorded and analysed with FloJo software v10. Gene Expression of NLRP3 via rtPCR was recorded in 10 patients and 10 controls at baseline and following LPS and melatonin treatment.

Results Granulocyte CD11b expression was lower in children with TBI compared to controls $(p=0.04)$ Both upregulated CD11b with LPS stimulation. Melatonin significantly decreased this LPS upregulation. There was no significant difference in baseline TLR4 expression between TBI and controls, but LPS upregulation of TLR4 was decreased by melatonin in the TBI cohort. Inflammasome was upregulated via NLRP3 expression in children with TBI compared to controls $(p=0.02)$. Melatonin significantly decreased LPS-induced upregulation of NLRP3 only in controls.

Conclusion Inflammation is altered in TBI compared to controls with altered responsiveness to melatonin treatment following LPS stimulation. The inflammasome is downregulated in children immediately following TBI. Selective inhibition of systemic inflammation targeting the inflammasome may have a future immunomodulatory role as a target in treating TBI.

\section{GP124 IL1- $\beta$ LEVELS AT PRESENTATION CORRELATE WITH SYMPTOM BURDEN AT 2 WEEKS IN PAEDIATRIC MILD TRAUMATIC BRAIN INJURY}

${ }^{1,2}$ Emer Ryan*, 'Lynne Kelly, ${ }^{1}$ Eimear Duff, ${ }^{3}$ Mark Bates, ${ }^{2}$ Turlough Bolger $1,2,4,5$ Eleanor Molloy. ${ }^{1}$ Discipline of Paediatrics, Department of Medicine, Trinity College, Dublin, Ireland; ${ }^{2}$ Tallaght University Hospital, Dublin, Ireland; ${ }^{3}$ Department of Surgery, Trinity Translational Medincine Institute, Dublin, Ireland; ${ }^{4}$ National Children's Research Centre, Dublin, Ireland; ${ }^{5}$ Coombe Women's Hospital, Dublin, Ireland

\subsection{6/archdischild-2019-epa.189}

Aims To evaluate components of the innate immune system, the inflammsome, in mild Traumatic Brain Injury (TBI) and the correlation with symptom burden 2 weeks from injury. We examine activation of the pathway at presentation, and 
also at 2 weeks from injury compared with healthy control children.

Methods Whole blood was sampled from children with mild TBI within 24 hours of injury and at two weeks from injury and compared to healthy paediatric controls at baseline. RNA was isolated and cDNA was synthesized. Gene Expression of NLRP3 and IL1 $\boldsymbol{\beta}$ via rtPCR was recorded in 22 patients and 5 controls at baseline and 15 patients at 2 weeks. The Post Concussive Symptom Inventory was administered at 2 weeks. A change from pre-injury baseline was recorded.

Results Inflammasome was upregulated via NLRP3 expression in children with TBI compared to controls across groups however this did not correlate with symptoms at 2 weeks. Higher IL-1 $\beta$ transcription levels at presentation were positively correlated by Pearson correlation $(\mathrm{p}=0.029)$ with higher symptom scores at 2 weeks.

Conclusion Inflammation is altered in TBI compared to controls The NLRP3 component of inflammasome while elevated does not correlate with symptom burden. $\mathrm{IL}=1 \boldsymbol{\beta}$ gene transcription does. IL-1 $\boldsymbol{\beta}$ holds promise in predicting symptom burden following mTBI. Selective inhibition of systemic inflammation targeting the inflammasome may have a future immunomodulatory role as a target in treating mTBI.

\section{GP125 MY LIFE, MY VOICE: TECHNOLOGY-ENHANCED QUALITY OF LIFE ASSESSMENT TOOL FOR CHILDREN WITH HYDROCEPHALUS}

1,2,3 Joy Tan*, ${ }^{3,1}$ John Caird, ${ }^{1,2}$ Alf Nicholson. ${ }^{1}$ Temple Street Children University Hospital, Dublin, Ireland; ${ }^{2} R C S I$, Dublin, Ireland; ${ }^{3}$ Beaumont Hospital, Dublin, Ireland

\subsection{6/archdischild-2019-epa.190}

Background To date, children with hydrocephalus continue to have a considerable long-term outcome. However, current literature on health-related quality of life (HRQOL) among children with hydrocephalus are limited. This serves a call for research to validate a suitable HRQOL for children with hydrocephalus measuring the physical, emotional, social and cognitive well being.

Objective To validate a technology-enhanced quality of life questionnaire (EITVAQ) as an effective assessment tool measuring the current well-being of a child with hydrocephalus (child-centred)

To adopt a technology-enhanced quality of life assessment tool (EITVAQ) into a phone/tablet application to gain widespread usage

To explore the use of technology to increase the learning potential and obtaining a new skill among children with hydrocephalus

Methods This is a prospective study which took place since January 2018 to January 2019.

This study received ethical approval All data was compiled and analysed using SPSS. The validation process involved: Content Validity, Feasibility and Reliability. A cohort of 70 healthy children from various primary and secondary schools participated as a control. Finally, a small cohort of children with hydrocephalus participated in technology-enhanced learning programme (TELP) to learn a new music skill and created a music video.
Results This study reports the result of the validation process. In total there were 132 participants. Content validity was achieved by the nominal group technique. In this study, technology enhanced quality of life questionnaire (EITVAQ) is a feasible tool, having a high satisfaction rate of $78 \%$, a response rate of $76.5 \%$ and an average completion time of 4 mins and 26 seconds. EITVAQ had a score of 0.92 (Cronbach Alpha). When comparing with the control, the two lowest scores were the social and emotional aspect. The two major differences in scores among both groups were the physical and cognitive well-being. All participants who participated in the TELP managed to complete the task learning the skills of creating a music video.

Conclusion EITVAQ, an interactive and child-friendly tool to assess quality of life is now validated. It aims to be used widely among children with hydrocephalus, providing a baseline assessment to allow us to understand more about a child's quality of life from their own perspective. This study concludes that technology has a huge potential in helping children with hydrocephalus and various neuro-disability to integrate into society.

\section{GP126 LARGE DELETIONS IN DMD GENE IS THE MOST PREVALENCE MUTATION IN RUSSIAN CHILDREN WITH DUCHENNE MUSCULAR DYSTROPHY}

Kirill Savostyanov*, Alexander Pushkov, Ludmila Kuzenkova, Natalya Zhurkova, Alexey Nikitin, Alexey Kurenkov, Bella Bursagova, Sergey Trufanov. FSAI National Medical Research Center for Childrens Health, Moscow, Russian Federation

\subsection{6/archdischild-2019-epa.191}

Background and objectives Duchenne muscular dystrophy (DMD) is a rare muscle disorder inherited by X-linked recessive type and affecting approximately 1 in 3,500 male births worldwide.

Patients and methods The study included 63 boys, aged from 6 months to 8 years with elevated creatinine phosphokinase (CPK), according to laboratory tests. After medical genetic counseling molecular genetic analysis was performed for all patients. The MLPA method was used to search for deletions and duplications in the $D M D$ gene, the analysis of point mutations was carried out by NGS, if the MLPA method did not reveal pathogenic variants. Sanger sequencing was used to validate mutations identified by the NGS.

Results Totally, in 39 patients we revealed different alterations in DMD gene. Among them 11 (28\%) had a point mutations. It was 4 nonsense, 4 missense, 2 splicing mutation and one single-nucleotide duplication. Five mutation were novel. They are splicing c.10798-2A>G, missense c.2288T $>A$ (p.Val763Asp) and c.3269A >T (p.Gln1090Leu), nonsense $c .858 T>G(p . T y r 286 X)$ and duplication c.8325dup (p.Gln2776Thrfs*6).The remaining $28(72 \%)$ patients had gross duplications $3(8 \%)$ and gross deletions $25(64 \%)$ in the $D M D$ gene. Interestingly, more than half of the patients had deletions in the region of exons 45-51 of the DMD gene.

Conclusion Our study showed that the most common cause of Duchenne dystrophy in Russian children are gross deletions of the $D M D$ gene, in particular deletions in the region of exons 45-51 occurring most frequently. 